\title{
Deformation Mechanisms of Polymer Thin Films by Simultaneous Kinetic Measurement of Microscopic Infrared Dichroism and Macroscopic Stress. Part III: Influence of Morphology on Molecular Orientation of Isotactic Polypropylene Films Subjected to Uniaxial Stretching
}

\author{
Yihu SonG ${ }^{\dagger}$, Koh-hei NITTA*, and Norio NEMOTO* ${ }^{*}$ \\ † Department of Molecular and Material Sciences, IGSES, Kyushu University, Hakozaki, Fukuoka 812-8581, Japan \\ $\$$ School of Materials Science, Japan Advanced Institute of Science and Technology, Ishikawa 923-1292, Japan \\ (Received : January 14, 2003)
}

\begin{abstract}
Molecular orientations of the crystalline and the amorphous phases are studied in relation to morphology using an instrument constructed for simultaneous kinetic measurement of microscopic infrared (MicIR) dichroism from a predetermined sampling area and macroscopic stress of isotactic polypropylene (iPP) thin film uniaxially stretched at a constant strain rate at room temperature. The morphology imposes important influences on profiles of MicIR dichroism as a function of stretching time and also on a relationship between the orientation functions, $f_{\mathrm{c}}$ and $f_{\mathrm{am}}$, of the crystalline and the amorphous phases. Linear relationships between $f_{\mathrm{c}}$ and $f_{\mathrm{am}}$ are found for the local areas without giant spherulite inclusions when $f_{\text {am }}<0.3 \sim 0.4$, suggesting the harmonious developments of amorphous chain deformation and lamellar orientation during the necking propagation. The proportionality constant between $f_{\mathrm{c}}$ and $f_{\mathrm{am}}$ is not universal but is strongly dependent on the morphology of the surrounding area where the local area under examination is located. The linear relationship between $f_{\mathrm{c}}$ and $f_{\mathrm{am}}$ is completely invalidated for describing the relationship between the amorphous chain deformation and the lamellar orientation inside the spherulites, which reveals that the deformation mechanism in the spherulite is different from that in the microcrystalline region.

Key Words: Isotactic polypropylene / MicIR dichroism / Morphology / Necking
\end{abstract}

\section{INTRODUCTION}

Inhomogeneous deformation is inevitable to semicrystalline polymers in which macromolecules are presented in such various levels of spatial arrangement as lamellae, lamellar clusters, spherulites and amorphous chains. There is a great interest in the plastic deformation over the past decades, ${ }^{1-22}$ ) making considerable progress in understanding the macroscopic mechanical behavior in relation to the microscopic structure evolution. However, it is realized only recently that the macroscopic stress-strain curves do not provide any correct description of the inhomogeneous deformation accompanied by neck propagation, which suggests that the measurement of both true stress and true strain in local area is crucial to understand the inhomogeneous deformation mechanism of semi-crystalline polymers. ${ }^{23-25)}$

* To whom correspondence should be addressed. E-mail: nemo@mm.kyushu-u.ac.jp

$\dagger$ Kyushu University

$\uparrow$ Japan Advanced Institute of Science and Technology
For illuminating the microscopic molecular deformation, meso-scale morphological evolution and the macroscopic mechanical behavior during the plastic deformation, the microscopic infrared (MicIR) dichroism method is unique among other scientific techniques used for morphological and structural detection of deformation. In an earlier paper, ${ }^{26)}$ we have shown that the necking causes a dominant molecular deformation within a pre-determined sampling area in an isotactic polypropylene (iPP) thin film with a smectic structure when it is uniaxially stretched at the room temperature. During the necking process, the molecular orientation function $f_{\mathrm{am}}$ in the amorphous phase closely exhibit the behavior predicted for the affine deformation up to a considerably large local stretching ratio of $\lambda \sim 4$ and furthermore the molecular orientations of the crystalline and the amorphous phases are interdependent so that a linear relationship between $f_{\mathrm{c}}$ and $f_{\text {am }}$ with a slope of 1.85 may hold for $\lambda<4$, beyond which the plastic deformation components become occasionally dominant in the local area under examination. ${ }^{27)}$ Here, $f_{\mathrm{c}}$ is the 
orientation functions in the crystalline phase. To examine the applicability of the linear relationship and consequently of the proportionality constant between $f_{\mathrm{c}}$ and $f_{\mathrm{am}}$, the molecular orientation in more inhomogeneous iPP film samples with various morphologies will be studied in this paper.

In Ref. 26, we have noticed that the molecular orientation is definitively related to the necking when the film is homogeneous, whereas the situation becomes complicated when the film contains a giant spherulite (GS). Because of the rigid nature of the GS, it can prevent the necking to some extent and leads to an inhomogeneous deformation of the microcrystalline region in the close vicinity of GS. In Ref. 26, we have presented the data in terms of orientation angles as a function of nominal strain, which is not enough for characterizing the deformation of a film with an inhomogeneous morphology. In this wok, we will apply the photogrammetry method developed in Ref. 27 to pursue the morphological evolution of a local small area along side the MicIR dichroism and the macroscopic stress measurements. Therefore, the second purpose of this work is to clarify the influence of the pre-existed morphology of the desired small local area on the necking propagation and the resultant molecular orientation. Experimental investigations are performed to the molecular orientation with three kinds of sampling area. The first one itself is apparently homogeneous but is located in the films with two different morphologies. The second one is deliberately arranged so as to contain a rigid domain that could not deform largely. The third itself is a rigid domain. Such arrangements are based on our observation that the deformation of the melt cast iPP films is completely inhomogeneous but shows morphology dependence. Some local domains with a high content of the crystalline phase are usually less deformed or could not be deformed even though the films are stretched to a macroscopic strain as large as 12 and above.

\section{EXPERIMENTS}

\subsection{Materials}

Isotactic polypropylene (iPP) with an average molecular weight $\mathrm{Mw}=3.7 \times 10^{5}$ was used. The iPP pellets were thermally compressed at $210^{\circ} \mathrm{C}$ for $5 \mathrm{~min}$ to form the films with an average thickness of about $30 \mu \mathrm{m}$, which were then annealed at two different conditions. When annealed at $135^{\circ} \mathrm{C}$ for $30 \mathrm{~min}$, the film (coded film-A) obtained was apparently homogeneous and only contained microcrystals of a size less than $10 \mu \mathrm{m}$ in diameter. When annealed at $145^{\circ} \mathrm{C}$ for $240 \mathrm{~min}$, the film (coded film-B) was found to contain giant spherulites with the $\alpha$ type of crystalline structure and a diameter of about $300 \mu \mathrm{m}$.

Crystal-like domains with irregular shapes and with a size from several microns to several decades of micron were found to be considerably abundant in both films, which may be aggregates of small crystallites. One of those crystal aggregates was carefully chosen in the film center before elongation as a mark of the sampling area to which the MicIR dichroism was sampled. Only data from the samples with a mark distinguishable up to the end of elongation were analyzed. The film-B contains one giant spherulite (GS) and its center was used as a mark when measuring the molecular orientations within GS or in a sampling area containing a GS.

\subsection{Apparatus and Method}

The setup details of the equipment were described in an earlier paper. ${ }^{28)}$ The kinetic measurement of $A C(t)=A_{\|}(t)-A_{\perp}(t)$ and $D C(t)=A_{\|}(t)+A_{\perp}(t)$ and the data analysis procedure were described elsewhere. ${ }^{26)}$ Here $A_{\|}(t)$ and $A_{\perp}(t)$ are the polarized spectra at stretching time $t$ with the IR radiation parallel and perpendicular to the draw axis, respectively.

The sample cell of the elongation device was mounted in the same compartment of the FT-IR system. The infrared or visible beam was irradiated directly on the film from the bottom, which allowed us to sample the infrared signal or to observe the mark by switching the view/detection selection optics. Because of the synchronized movement of the two clamps to the two opposite directions from the center of the film and because of the low elongation speed, it was convenient to adjust the mark always to the fixed laboratory coordinates under the desired viewing field at a time interval of 3 minutes whenever the sampling area did not stay in its original position. Such adjustment made it possible to sample the MicIR dichroism from the same area as accurately as possible.

The film was cut to rectangular-shaped specimens with a size of $10 \times 3 \mathrm{~mm}^{2}$ (length $\times$ width), which were fastened between the two mechanical clamps and were stretched at 30.0 $\pm 0.1^{\circ} \mathrm{C}$ with a constant macroscopic strain rate of $Y=V_{\mathrm{E}} / L_{0}=$ $0.066 \mathrm{~min}^{-1}$. Here, $V_{\mathrm{E}}$ and $L_{0}$ are the stretching speed and the initial film length in the range of $0.5 \mathrm{~mm} \sim 2.0 \mathrm{~mm}$, respectively.

The viewing field was from $600 \times 600 \mu \mathrm{m}^{2}$ to $100 \times 100 \mu \mathrm{m}^{2}$, depending on the local morphology of the desired sampling area. The parameters of the spectrometer were the same as described in the earlier paper. ${ }^{26)}$ The Hermans orientation 
function $f(t)$ for a particular absorption band can be derived as

$$
f(t)=\frac{2 A(t)}{3-A(t)} \frac{R_{0}+2}{R_{0}-1}
$$

where $R_{0}=2 \cot ^{2} \psi$ is the perfect dichroic ratio of a transition moment making an angle $\psi$ with the direction of the molecular main chain axis and $A(t)=A C(t) / D C(t) . f_{\mathrm{c}}$ was determined from the $998 \mathrm{~cm}^{-1}$ band, ${ }^{29)}$ while $f_{\text {am }}$ was derived from the 973 $\mathrm{cm}^{-1}$ and $998 \mathrm{~cm}^{-1}$ bands based on a two-phase model 29-31) when the sampling area did not contain a GS. On the other hand, $f_{\text {am }}$ within a GS was determined from the $2723 \mathrm{~cm}^{-1}$ band ${ }^{30)}$ due to the invalidation of the two-phase model.

A method of photogrammetry was employed for in-situ observation of the morphology within the sampling area. A digital camera equipped with a microscopical lens was connected with the ocular of the IR microscope and micrographs of the sampling area were recorded at selected $t$

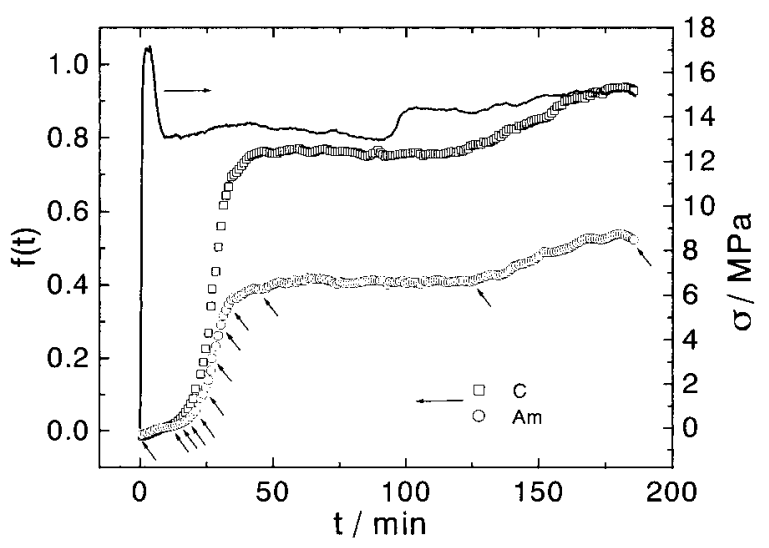

(a)

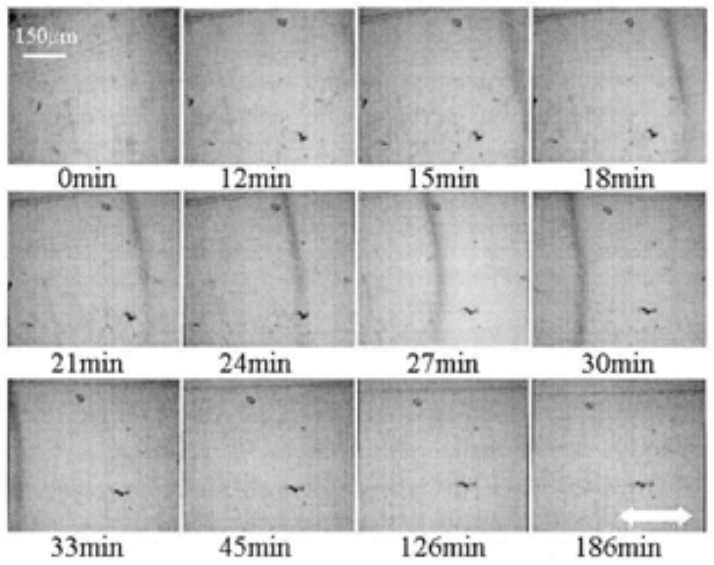

(b)

Fig.1 (a) Time profiles of the orientation functions $f_{\mathrm{c}}$ and $f_{\mathrm{am}}$ in the crystalline (C) and the amorphous (Am) phases from a predetermined sampling area of $600 \times 600 \mu \mathrm{m}^{2}$ and the macroscopic stress $\sigma$ of the film-A; (b) Morphologies of the sampling area at selected stretching time $t$. during elongation.

Morphologies of interested local area before and after uniaxial stretching without loading were observed using a polarizing optical microscope (Nikon, Poh22326) with a sensitive colour plate.

\section{RESULTS AND DISCUSSION}

\subsection{Dichroism Profile and Morphology Evolution in the Film-A}

$f_{\mathrm{c}}$ and $f_{\mathrm{am}}$ from a sampling area with a size of $600 \times 600 \mu \mathrm{m}^{2}$ are shown in Fig.1(a) as a function of $t$ together with the macroscopic nominal stress $\sigma$ profile. Corresponding micrographs of the sampling area are given in Fig.1 (b). The sampling area contains several crystal aggregates with a size of $10 \mu \mathrm{m} \sim 30 \mu \mathrm{m}$, which are less deformed during the entire elongation of the film. The film yields at a yielding time $t_{\mathrm{y}}=$

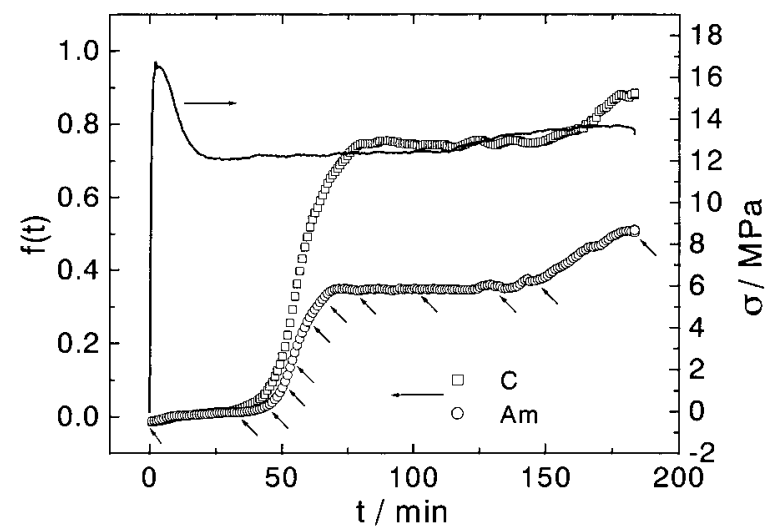

(a)

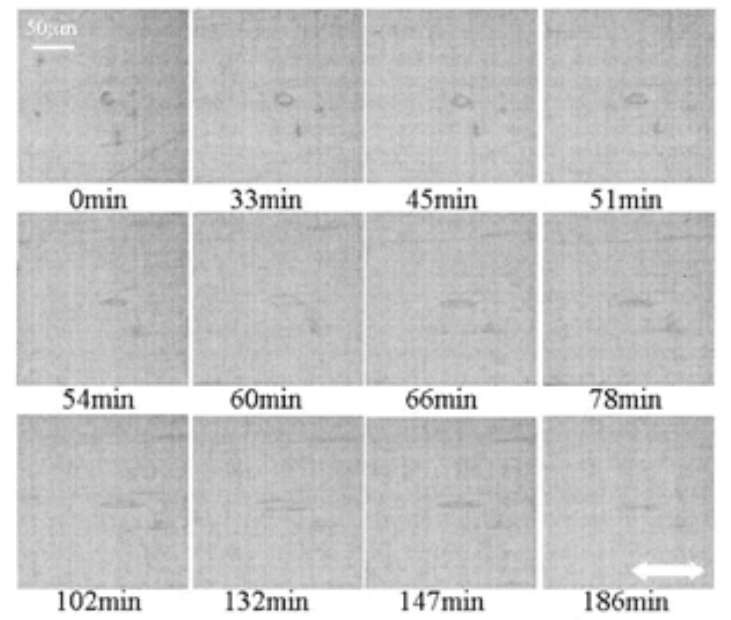

(b)

Fig.2 (a) Time profiles of $f_{\mathrm{c}}$ and $f_{\mathrm{am}}$ from a pre-determined sampling area of $200 \times 200 \mu \mathrm{m}^{2}$ and $\sigma$ of the film-A; (b) Morphologies of the sampling area at selected stretching time $t$. 
2.4 min followed by a strain softening and a further cold drawing. However, molecules start to orient markedly later than the end of the strain softening. As is clearly seen from Fig.1(b), a deformation band denoting the necking front propagates gradually through the sampling area from $t=15$ $\min$ to $t=33 \mathrm{~min}$ and during this time interval sharp increases in $f_{\mathrm{c}}$ and $f_{\mathrm{am}}$ undoubtedly occur. Molecules in the sampling area are oriented slightly when $t<15 \mathrm{~min}$ due to a small deformation in the outer section that is not suffered from the necking yet. After the necking passes through, the sampling area is less deformed from $t=45 \mathrm{~min}$ to $t=126 \mathrm{~min}$, resulting in the plateau for $f_{\mathrm{c}}$ and $f_{\mathrm{am}}$, though a strain hardening is observed from $t=90 \mathrm{~min}$ to $t=100 \mathrm{~min}$. The further molecular orientations at $t>126 \mathrm{~min}$ are mainly induced by a localized deformation in the sampling area that is already located at the necked down region, i.e. the neck entity, or it may be related to the apparently homogeneous deformation in the whole length scale of the sample after the necking is finished. This result reconfirms a previous conclusion ${ }^{26,27)}$ that the necking

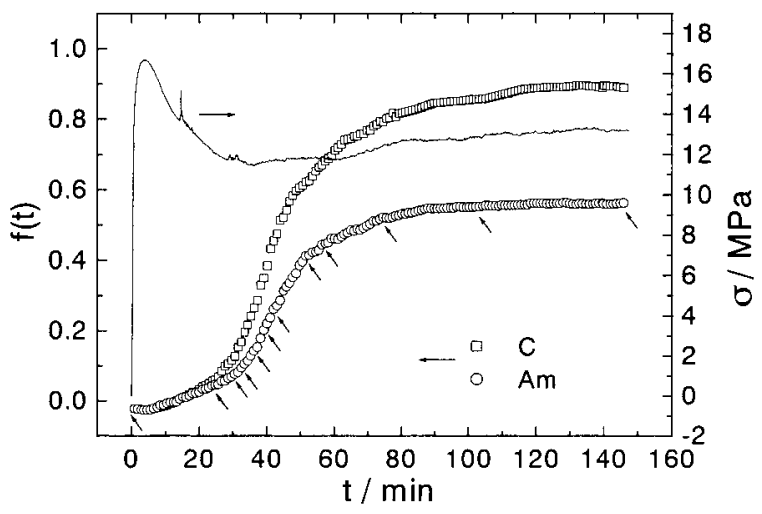

(a)

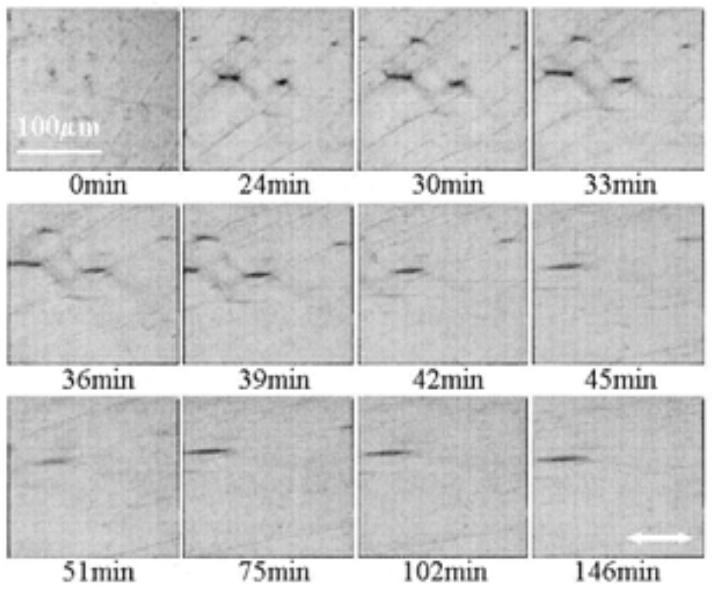

(b)

Fig.3 (a) Time profiles of $f_{\mathrm{c}}$ and $f_{\mathrm{am}}$ from a pre-determined sampling area of $200 \times 200 \mu \mathrm{m}^{2}$ and $\sigma$ of the film-B without giant spherulite inclusions in the stretching section; (b) Morphologies of the sampling area at selected stretching time $t$. propagation induces an exclusive molecular orientation as a concomitant of local deformation in the activated neck shoulder, which was realized by Onogi ${ }^{32)}$ and Siesler ${ }^{29,33)}$ past decades earlier. From the movement of the neck front, the necking propagation speed (relative to the marks of the sampling area) is estimated as $V_{\mathrm{NP}}=33 \mu \mathrm{m} \mathrm{min}$.

Figure 2 shows the results from a sampling area of $200 \times 200$ $\mu^{2}$ in another sample in which the deformation band is not distinguishable. It is seen that $f_{\mathrm{c}}$ and $f_{\text {am }}$ have the similar profiles to those in Fig.1, except for the onset time of the molecular orientation is postponed until $t=42 \mathrm{~min}$ due to the distance between the sampling area and the neck initiation position larger than that of the sample in Fig.1. The rapid increases in $f_{\mathrm{c}}$ and $f_{\mathrm{am}}$ is consistent with an increasing deformation of a crystal aggregate in Fig.2(b).

\subsection{Dichroism Profile and Morphology Evolution in Microcrystalline Region of the Film-B}

In order to make a comparison of molecular orientations with the film-A, we first study a sample of the film-B without GS inclusions in the elongation section. The $\sigma$ profile in Fig.3(a) shows a sharp stress peak at $t=15$ min appeared during the strain softening whose time interval is obviously larger than that of the film-A. It seems that only some of the imperfect crystals deform plastically at the yielding point and initiate the necking, whereas some crystals with a high order are further destructured during the strain softening that gives the second $\sigma$ peak. $f_{\mathrm{c}}$ and $f_{\text {am }}$ in Fig.3(a) might be divided into three regions due to the different deformations of the sampling area in the outer section, the neck shoulder and the neck entity. The first region is related to the same mechanism as those observed in the film-A as the slight deformation of the outer section after the film is yielded. The watershed between the second and the third regions is not as distinct as the MicIR dichroism of the film-A, suggesting that the shape of the neck shoulder is not constant but the length increases during passage through the sampling area. The lengthening of the neck shoulder may be involved in a determinate plastic deformation of the crystalline phase. It is of importance to find that molecules are deformed slightly in the third region after the sampling area is located at the neck entity, which is consistent with local deformation in the sampling area as denoted by the increasing aspect ratio of a crystalline aggregate in Fig.3(b). This result together with that in Fig.2(b) may need a correction of the previous viewpoint that the neck simply propagates through the specimen without further stretching of the material within the earlier necked region. ${ }^{34-36)}$ 
It is an experimental observation that one GS in the elongation section of a film may influence the $\sigma$ profile considerably. The deformation of the sample containing one GS is likely to give two kinds of initial necking that are far from or very close to the GS, respectively. In the first case, the spherulite boundary possesses a moderate mechanical strength so that the GS may prevent the necking passage. It takes a long time for the microcrystalline region in the vicinity of the GS to develop a molecular orientation. In the second case, the stack defects at the spherulite boundary facilitate the yielding and the resultant necking leads molecules in the surrounding microcrystalline region to orient from the earlier stage of elongation. In fact, this is only a very coarse classification because it is really a problem to make a judgment of the distance between the neck initiation position and the film center (or the spherulite center).

Using three kinds of arrangement of the sampling area of $100 \times 100 \mu \mathrm{m}^{2}$ and the isolated GS, the molecular orientations and the morphology evolution are given in Figs.4, 5 and 6, respectively. In Fig.4 where the sampling area is located next to the spherulite polar axis $300 \mu \mathrm{m}$ far away from the GS boundary, we obtain the first case of the initial necking. The dramatic molecular orientation starts from $t=100 \mathrm{~min}$, as evident by a deformation band observed at $t=102 \mathrm{~min}$, hereafter the crystal aggregate is destroyed to small pieces that remain up to the end of elongation. The two broad stress peaks in Fig.4(b) come from the stress concentration at the two polar points of the GS followed by the boundary cracking as shown in the polarized micrographs. It is clear that there is no deformation of the sampling area at $t<100$ min after a very small molecular orientation occurred before the yielding where the film is deformed homogeneously. After $t>110 \mathrm{~min}$, a slow molecular orientation is observed even after the secondary boundary cracking occurs. The plastic deformation allows the coexistence of the molecular orientation and the cracking in the entire length scale of the sample.

In Fig. 5 where the sampling area is located next to the spherulite equatorial axis, we obtain the second case of the initial necking. The dramatic molecular orientation starting from $t_{\mathrm{y}}=4 \mathrm{~min}$ is induced mainly by a shearing band across the sampling area, which is visible from $t=9 \mathrm{~min}$ to $t=24 \mathrm{~min}$. After the shearing band passes through, molecules are oriented continuously via a different deformation mode until $t=68 \mathrm{~min}$ for the amorphous phase and $t=83 \mathrm{~min}$ for the crystalline phase. The molecular orientation is decelerated from $t=40 \mathrm{~min}$ due to the lengthening of the neck shoulder. The sampling area is moved away from the spherulite equatorial axis (Fig.5a) due to the plastic flow induced by an asymmetrical boundary cracking. Moreover, the asymmetrical cracking causes a simultaneous rotation of the GS with the GS plastic
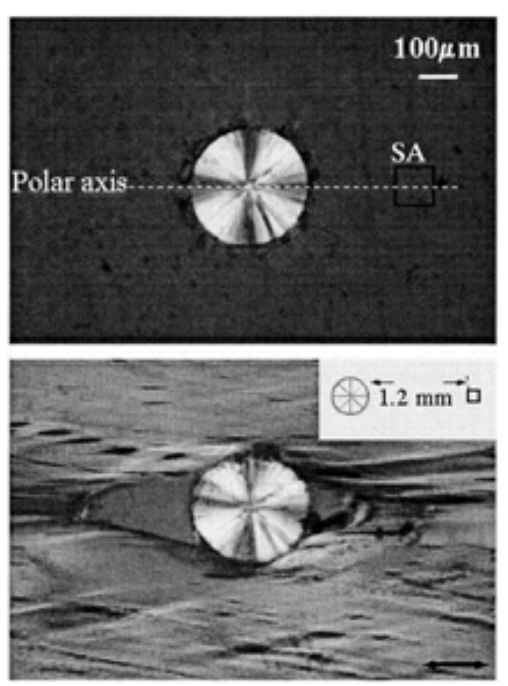

(a)

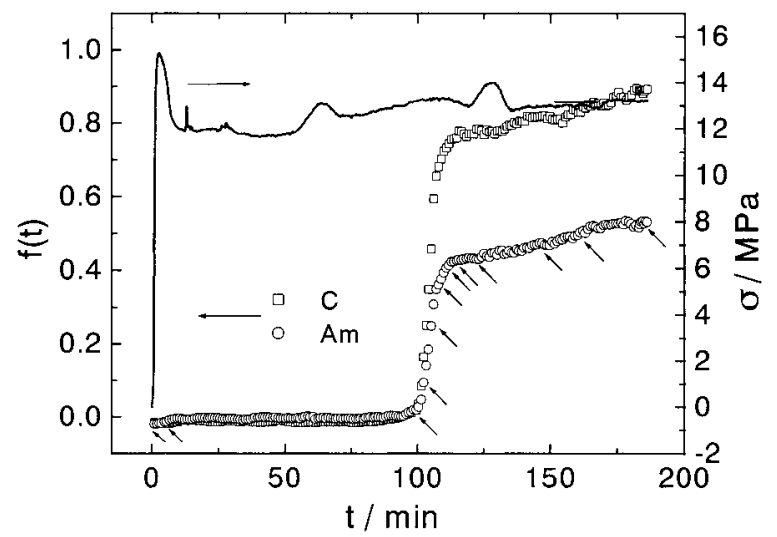

(b)

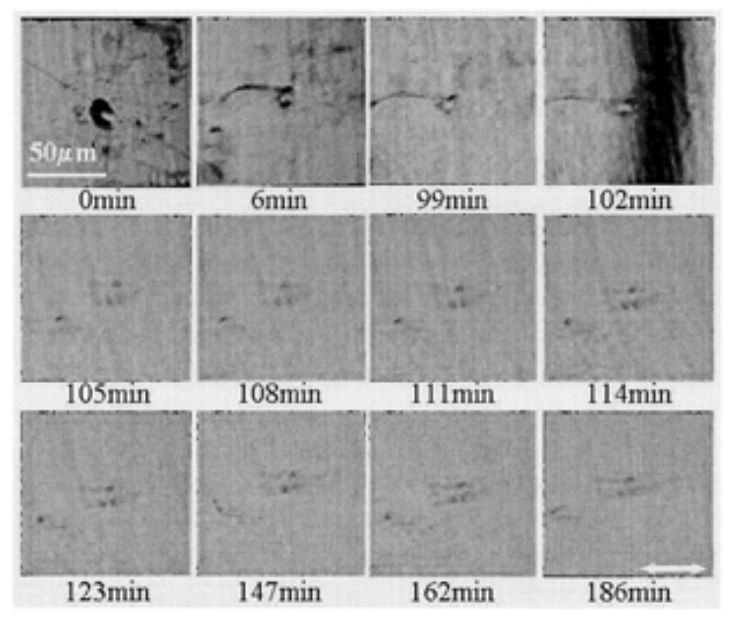

(c)

Fig.4 (a) Micrographs around a giant spherulite at macroscopic draw ratios of 1 (top) and 13 (bottom) of the film-B with an arrangement of the sampling area (SA) of $100 \times 100 \mu \mathrm{m}^{2}$ next to the spherulite polar axis; (b) Time profiles of $f_{\mathrm{c}}$ and $f_{\text {am }}$ and $\sigma$ of the film-B; (c) Morphologies of the sampling area at selected stretching time $t$. 
deformation.

In Fig.6 where the sampling area is located with its center 30 $\mu \mathrm{m}$ away from the spherulite equatorial axis, the time profiles of dichroism are quite different from those in Figs. 4 and 5. The

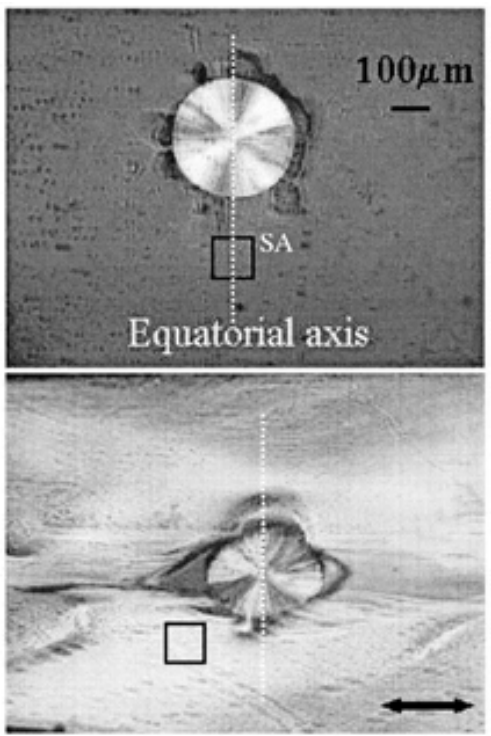

(a)

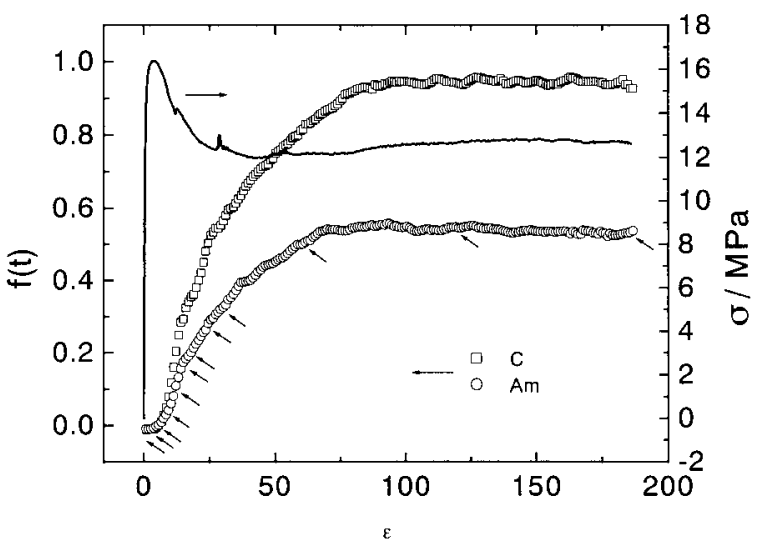

(b)
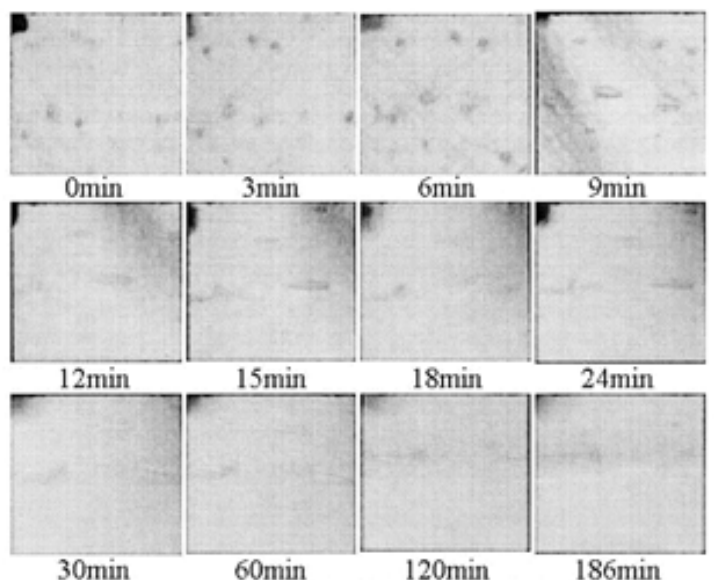

(c)

Fig.5 (a) Micrographs around a giant spherulite at macroscopic draw ratios 1 (top) and 13 (bottom) of the film-B with an arrangement of the sampling area (SA) of $100 \times 100 \mu \mathrm{m}^{2}$ next to the spherulite equatorial axis; (b) Time profiles of $f$ and $f_{\text {am }}$ and $\sigma$ of the film-B; (c) Morphologies of the sampling area at selected stretching time $t$. much slower molecular orientation in comparison with the other specimens is explained by a considerably slow propagation of the shearing band (Fig.6c). The boundary cracking causes a long distance migration of the sampling area
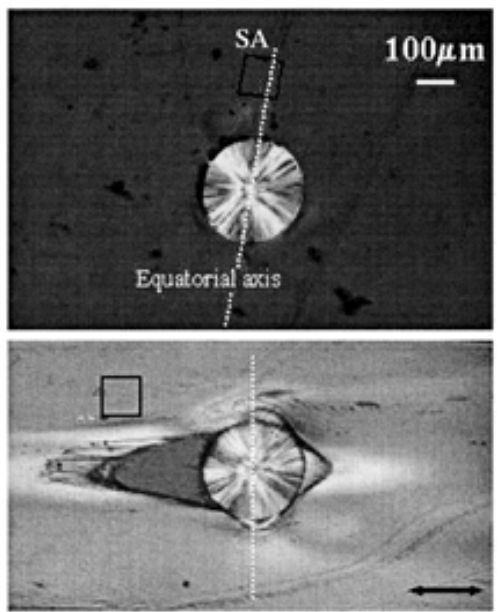

(a)

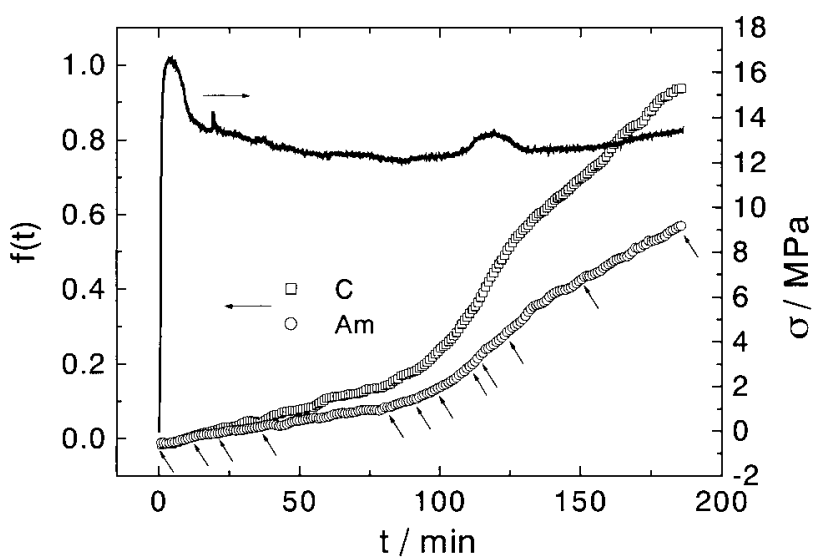

(b)
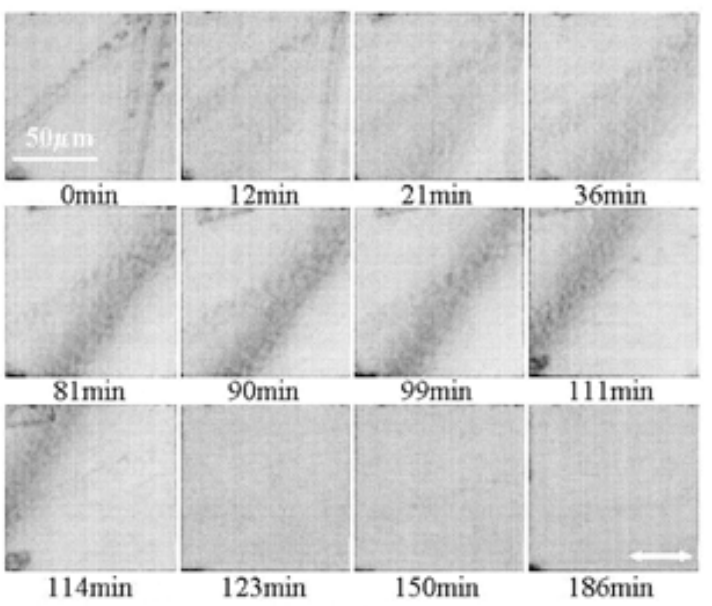

(c)

Fig.6 (a) Micrographs around a giant spherulite at macroscopic draw ratios of 1 (top) and 13 (bottom) of the film-B with an arrangement of the sampling area (SA) of $100 \times 100 \mu \mathrm{m}^{2}$ with its center deviating $30 \mu \mathrm{m}$ from the spherulite equatorial axis; (b) Time profiles of $f_{\mathrm{c}}$ and $f_{\mathrm{am}}$ and $\sigma$ of the film-B; (c) Morphologies of the sampling area at selected stretching time $t$. 
along the draw axis and also slows the molecular orientation. It is seen that the molecular orientation is decelerated after the boundary cracking at $t=120 \min$ whereas $f_{\mathrm{c}}$ and $f_{\text {am }}$ eventually reach the values comparable with those in Figs. 4 and 5 at a macroscopic draw ratio 13 of the film.

The results in Figs.4, 5 and 6 suggest that the plastic deformation could not be simply viewed as the equivalent of the plastic flow. The plastic flow is not observed in all the sampling areas and the GSs tested, even through they are moved continuously along the draw axis during the elongation. On the other hand, the localized plastic flow may occur accompanied by the microcracking, which gives a hindrance to the plastic deformation and the molecular orientation. The quite different MicIR dichroism profiles come from the influence of the plastic flow on the deformation fashion of the microcrystalline region located at different positions of the GS with reference to the draw axis. In Fig.5, the increased distance between the GS and the sampling area

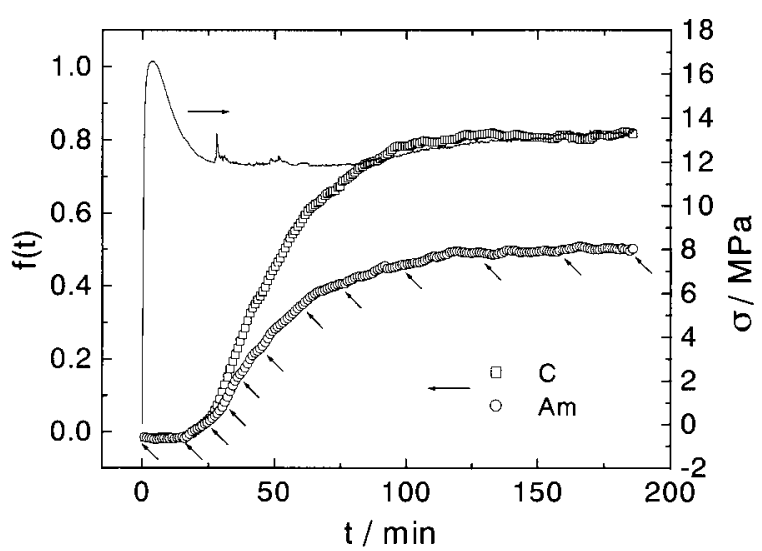

(a)
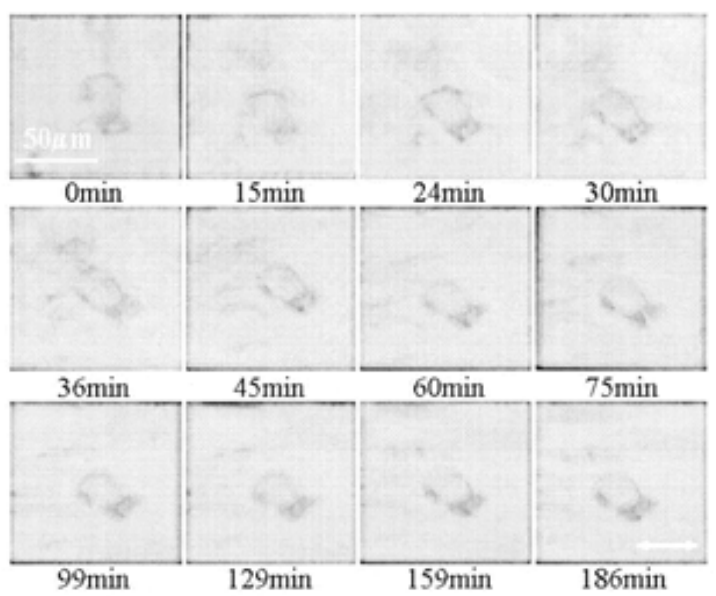

(b)

Fig.7 (a) Time profiles of $f_{c}$ and $f_{\text {am }}$ from a sampling area of $100 \times 100$ $\mu \mathrm{m}^{2}$ containing a hard crystalline aggregate with an area percentage of $9 \%$ and $\sigma$ of the film-B; (b) Morphologies of the sampling area at selected stretching time $t$. results in that the plastic flow could not influence the deformation of the sampling area. Here, only the necking is the reason of the very fast molecular orientation. On the other hand, the molecular orientation in the local area next to the GS equatorial axis occurs simultaneously with the plastic flow, which makes the contribution of the necking less important at the later stage.

\subsection{Influence of Rigid Microcrystalline Domain on Dichroism Profile}

The aforementioned dichroism profiles are obtained from the deformation of the sampling areas with an apparently homogeneous morphology, i.e., the visible crystal aggregates are either small enough compared with the sampling area or could be partially destroyed after the necking passage. There also exist some mechanically stable crystal aggregates in both films, an extreme example being the GS in the film-B. The mechanically stable crystal aggregates without polarized pattern of spherulite are called rigid microcrystalline domain

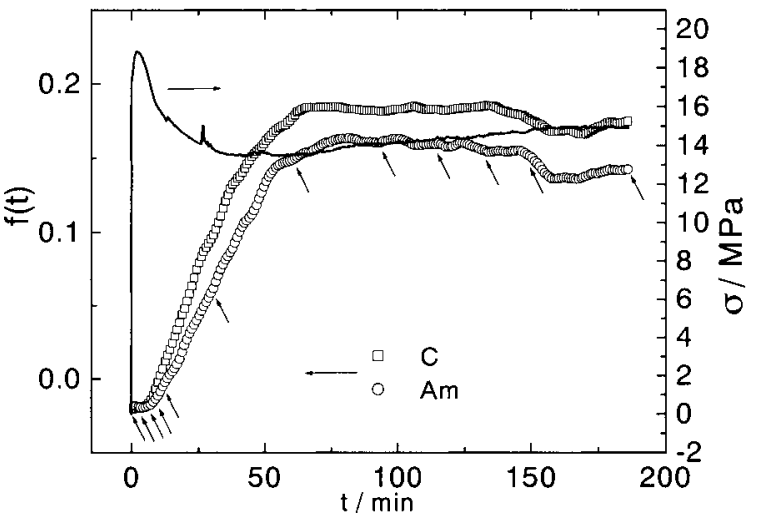

(a)

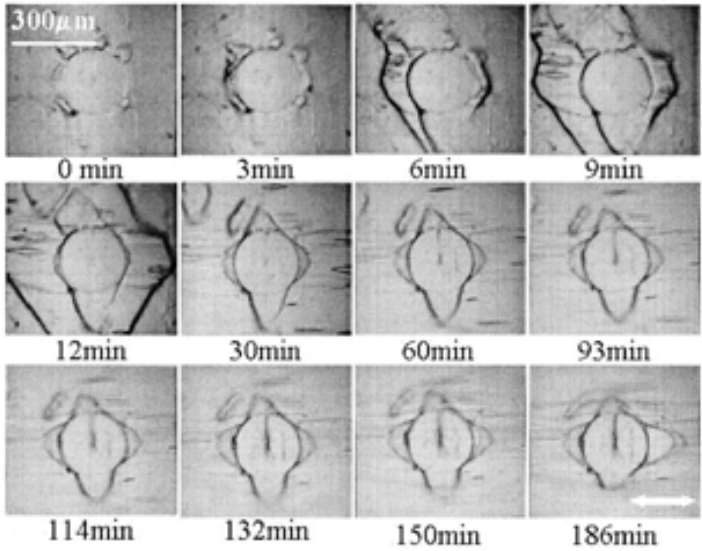

(b)

Fig. 8 (a) Time profiles of $f_{c}$ and $f_{\text {m }}$ from a sampling area of $600 \times 600$ $\mu \mathrm{m}^{2}$ containing a giant spherulite with an area percentage of $16 \%$ and $\sigma$ of the film-B; (b) Morphologies of the sampling area at selected stretching time $t$. 
(RMD) here so as to distinguish it from the GS. To understand the influence of the morphological inhomogeneities on the localized plastic deformation, we investigate the molecular orientations within the sampling areas containing two kinds of rigid domains, i.e., RMD and GS.

When a sampling area of $100 \times 100 \mu \mathrm{m}^{2}$ contains a RMD with an area percentage of $9 \%$, the necking passage results in the $f_{\mathrm{c}}$ and $f_{\mathrm{am}}$ values lower than those in the sampling areas with an apparently homogeneous morphology due to the resistance of the RMD to the local deformation, as shown in Fig.7. Morphology observation shows that the RMD under examination is embedded as an island in a highly deformed matrix, whereas other crystal aggregates become ellipses with their long axes parallel to the draw direction. There is no

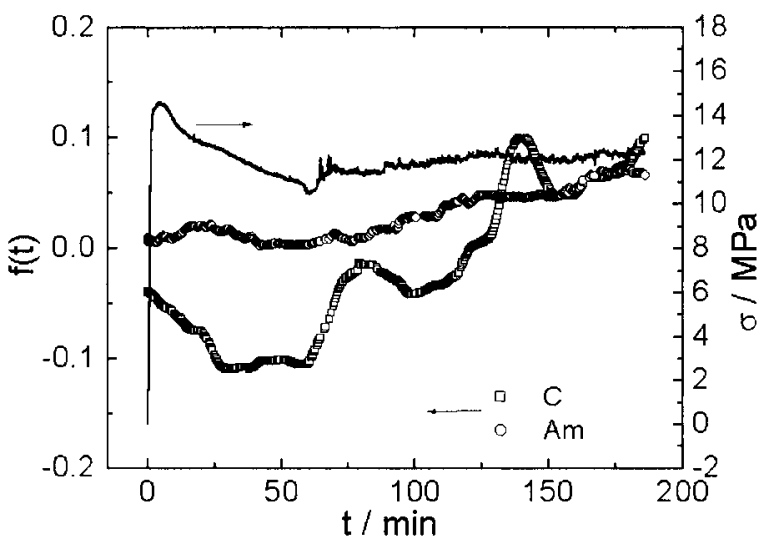

(a)
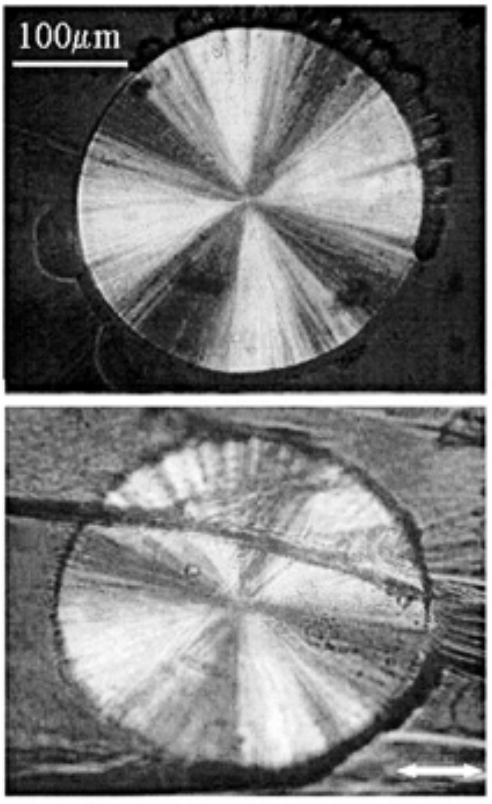

(b)

Fig.9 (a) Time profiles of $f_{\mathrm{c}}$ and $f_{\text {am }}$ within a giant spherulite using a square-shaped sampling area with the side length as same as the spherulite diameter of $270 \mu \mathrm{m}$ and $\sigma$ of the film-B; (b) Morphologies in the vicinity of the giant spherulite before (up) and after elongation (bottom) cracking occurred at the RMD boundary, suggesting a moderate interfacial strength. The low $f_{\mathrm{c}}$ shown at the neck entity in Fig. 7 may be due to the presence of the RMD in the sampling area, but this does not apply for $f_{\mathrm{am}}$. The deformation of the surrounding microcrystalline region forces some of the amorphous chains in the hard domain to orient whereas the lamellar framework impedes the orientation of the other tie chains.

In another sampling area of $600 \times 600 \mu \mathrm{m}^{2}$ containing a GS with an area percentage of $16 \%$, the averaged molecular orientations are considerably lower than those in Fig.7 after the necking passage through, as shown in Fig.8. The necking occurs initially at the GS boundary whereas its passage leaves two undeformed small areas next to the GS equatorial plane. It is obvious that the GS prevents the closely surrounding microcrystalline region from large deformation. The boundary cracking may also result in a reverse molecular orientation as shown by the decrease in $f_{\mathrm{c}}$ and $f_{\text {am }}$ from $t=136 \mathrm{~min}$ to $t=160$ min after the orientation plateau. The crystalline framework within a GS can resist the elongational stress effectively through slight lamellar rearrangement as revealed by the vertical deformation bands in the GS.

\subsection{Molecular Orientation within Giant Spherulite}

Spherulites are aggregates of anisotropic lamellae emanating from the common center nucleus. In an $\alpha$ spherulite of iPP, the chain $c$-axis of radial lamella may align in any direction perpendicular to radius, which, together with the approximately perpendicular arrangement of the tangential lamella, ${ }^{37)}$ should result in an approximately random orientation of the lamellar $c$-axis from the MicIR dichroism before stretching the film. Slight deviation from zero is usually observed for $f_{\mathrm{c}}$ because of the topological anisotropy of an oblate ellipsoidal shape of the GSs tested.

The GSs in the film-B are large enough for the direct MicIR dichroism study of the molecular orientation within them at low elongation speeds. An isolated GS deforms very slightly compared with the microcrystalline region, whereas the deformation is always nonaffine, plastic and irreversible almost occurring simultaneously with the boundary cracking. Figure 9 gives a typical result of MicIR dichroism sampled from a whole GS that enters in the neck at the early stage of elongation. Note that the lamella-like structure (the growing front during annealing) located at the boundary before elongation is transformed to the fibrous structure lying on the GS surface during elongation. The content of this fibrous structure is too small to give an influence on the measurement 
of the molecular orientations within the GS.

Different from the microcrystalline region, the orientations of amorphous chains and lamellae are locally dependent on their positions within the spherulite with respect to the draw axis. Several orientation mechanisms such as lamella tilting, slipping, shearing and fragmentation may occur concurrently in a local area and vary with respect to the deformation details of both the surrounding microcrystalline region and the spherulite, which is well outlined by $f_{\mathrm{c}}$ in Fig.9. We can distinguish that an overall parallel lamellar $c$-axis orientation occurs from $t=60 \mathrm{~min}$, where a macroscopic stress rise due to the stress concentration on the GS is observed. At higher strains, $f_{\mathrm{c}}$ does not increase monotonously but fluctuates obviously due to the lamellar relaxation after the boundary damage and the further lamellar rearrangement. Notwithstanding the vivid lamellar orientation, the amorphous chains within the rigid lamellar framework are oriented slightly toward the draw axis. The MicIR method does not provide data sufficient enough for revealing the complicated molecular orientation related to the plastic deformation of the GS. Thus it seems necessary to investigate the molecular deformation of the GS using the other powerful techniques along with the MicIR method.

\subsection{Relationship between $f_{\mathrm{c}}$ and $f_{\mathrm{am}}$}

Semi-crystalline polymers contain lamellae or crystallites with a typical thickness of about $10 \mathrm{~nm}$, which are separated by amorphous regions in the same size of order. The relatively flat lamellae can form bundles, stacks, or spherulites. A complex morphology must exist in a spatial scale of $100 \mu \mathrm{m}$, which determines the microscopic deformation of lamellae and amorphous chains from the MicIR dichroism profiles, the meso-scal morphology evolution from microscope observation and the macroscopic mechanical behavior.

It is shown that the propagation of the necking through a pre-determined sampling area with an apparently homogeneous morphology gives a preferred molecular orientation in both the film-A and the film-B. If the film-B contains an isolated GS, the molecular orientations in the surrounding microcrystalline region become complicated due to the dependence of the shear and the extension modes of deformation on the position of the observation area relative to the GS and, of course, the resistance of the GS to the necking as well as the consequential boundary cracking.

Microstructure changes associated to the plastic deformation have been documented for a long time. ${ }^{1-22,38-42)}$ Several microscopic deformations of importance are classified as deformation of the amorphous phase via interlamellar shear and separation, lamellar rotation and elastic deformation via stress transfer by tie-molecules, and plastic deformation of the crystalline phase via slip and dislocation. Interlamellar shear and separation are dominant when lamellar normals make an angle of $45^{\circ}$ and $0^{\circ}$ with respect to the draw axis and both are responsible for the deformation of amorphous chains. Lamellar rotation, being the most important contributor to the time evolution of $f_{c}$, occurs alongside interlamellar shear and separation so as to facilitate the further deformation of constraint amorphous chains. These deformation components must be dependent on the stack details of amorphous chains and lamellae, which, unfortunately, could not be distinguished from each other by the in-situ measurement of MicIR dichroism and morphology evolution. In fact, the MicIR dichroism is a result of all existing deformation modes in the sampling area.

After a larger number of measurements with well-defined conditions, we eventually realized that it is out of the question to discuss the physical meaning of the time profiles of the obtained IR dichroism. The above qualitative analyses of the molecular orientation combining with the $\sigma$ profile and the morphology evolution of the sampling area are somewhat reasonable, while they only reveal the complexity and are far from the nature of the plastic deformation. As we have seen, the plastic deformation is a multivariable process involved in the crystallinity, the crystal structure and the morphology around the sampling area and the necking as well as the microcracking. It is thus reasonable to discard the macroscopic variables of deformation (time, stress and strain) and to employ some microscopic variables suitable for understanding the underlying mechanism of the plastic deformation.

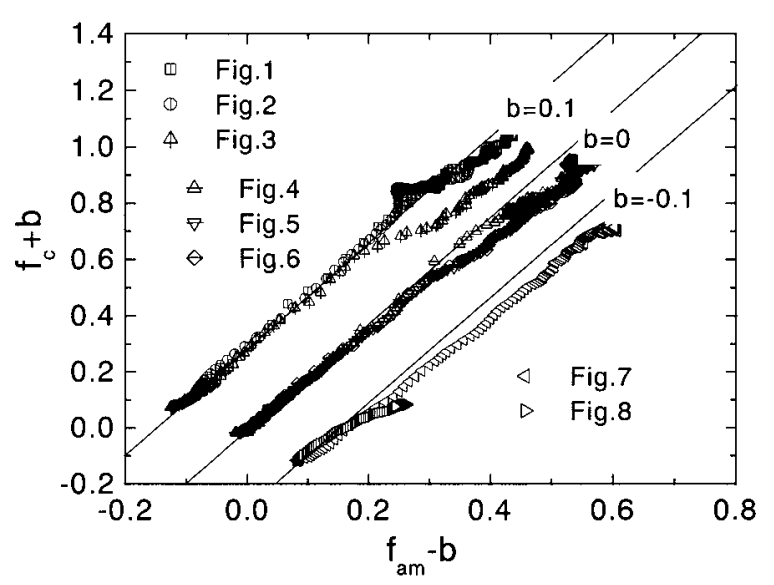

Fig.10 Relationship between $f_{\mathrm{c}}$ and $f_{\mathrm{am}}$. Three parallel lines with a slope of 1.85 are shown for guide of eyes. 
The results from Figs. 1 to 8 are re-plotted in Fig. 10 by means of $f_{\mathrm{c}}$ against $f_{\mathrm{am}}$ which reflects a relationship between the lamellar orientation and the amorphous chain deformation. Linear relationships are found to exist up to $f_{\mathrm{am}}=0.3 \sim 0.4$, except for the specimen in Fig.8 containing one GS in the sampling area. The influence of initial morphology is revealed from the different slopes. A slope of 1.85 in both the film-A and the film-B is independent of the size of the sampling area and the position of the initial necking. The difference in the results from the sampling area in the vicinity of a GS is considerably small up to $f_{\mathrm{am}}=0.3$, whereas the data fitting reveals the linear slopes of 1.85 and 1.70 when the observation area is located along the spherulite polar and the equatorial axes, respectively. The increased contribution of the shearing deformation seems to weaken the lamellar orientation slightly. In the sampling area containing a RMD, the linear relationship is still applicable but the slope is reduced to 1.60 due to the different restrictions of the RMD on the amorphous chain deformation and the lamellar orientation within it. On the other hand, the linear relationship is invalidated in the sampling area containing a GS because of the large inhomogeneous deformation around the GS (Fig.8) and the indefinite relationship between $f_{\mathrm{c}}$ and $f_{\text {am }}$ inside the GS (Fig.9).

The linear relationship between $f_{\mathrm{c}}$ and $f_{\mathrm{am}}$ when $f_{\mathrm{am}}<0.3 \sim 0.4$ in an apparently homogeneous local area suggests that the lamellar rotation cooperates with the lamellar shearing and by separation so as to harmonize the lamellar orientation with the deformation of amorphous chain during the necking initiation and propagation where the plastic deformation modes are not dominant. $f_{\mathrm{c}}$ versus $f_{\mathrm{am}}$ deviates from the linear relationships by an enhanced contribution of lamellar slip and dislocation and by the microcracking at the interface of lamellae and amorphous chains, which may occur at the neck entity or during the lengthening process of the neck shoulder.

It is shown that there is no universal value of the proportionality constant describing the relationship between $f_{\mathrm{c}}$ and $f_{\text {am }}$ when $f_{\text {am }}<0.3 \sim 0.4$ in the apparently homogeneous microcrystalline region. This value is also influenced by the morphology in the vicinity of the sampling area, other than the morphology of the sampling area itself. The morphology inhomogeneity of pre-existence is an essential factor that determines the necking initiation and propagation, the molecular orientations and the microcracking. The linear relationship is completely invalidated for describing the amorphous chain deformation and the lamellar orientation with the spherulites. The results reveal the completely different deformation mechanisms in the microcrystalline region and the spherulite.

\section{Acknowledgments}

This work has been partially supported by the Grant-in-Aid for the Scientific Research from Ministry of Education, Culture, Sports, Science and Technology, Japan (No. 10305070) and one of the authors, Y. Song, is grateful to JSPS for the Grant-in-Aid for JSPS Fellows relating to JSPS Fellowship for Foreign Researchers (No. 12000317).

\section{REFERENCES}

1) Lu J, Ravi-Chandar K, Intern J Solids Struc, 36, 391 (1999).

2) Tomita Y, Intern J Mechanical Sci, 42, 1455 (2000).

3) Ferreiro V, Pennec Y, Seguela R, Coulon G, Polymer, 41, 1561 (2000).

4) Castelein G, Coulon G, G'Sell C, Polym Eng Sci, 37, 1694 (1997).

5) Li JX, Cheung WL, Chan CM, Polymer, 40, 3641 (1999).

6) Flory PJ, Yoon DY, Nature, 272, 226 (1978).

7) Gent AN, Madan S, J Polym Sci Polym Phys Ed, 27, 1529 (1989).

8) Shadrake LG, Guiu F, Phil Mag, 34, 565 (1976).

9) O'Kane WJ, Young RJ, Ryan AJ, J Macromol Sci-Phys B, 34, 427 (1995).

10) Crist B, Fisher CJ, Howard PR, Macromolecules, 22, 1709 (1989).

11) O'Kane WJ, Young RJ, J Mater Sci Lett, 14, 433 (1995).

12) Hay IL, Keller A, Kolloid Z, 204, 43 (1965).

13) Hay IL, Keller A, J Mater Sci, 1, 41 (1966).

14) Hay IL, Keller A, J Mater Sci, 2, 538 (1967).

15) Young RJ, Mater Forum, 11, 210 (1988).

16) Krupenkin TN, Taylor PL, Macromol Theory Simula, 7, 119 (1998).

17) Karger-Kocsis J, Moskala EJ, Shang PP, J Thermal Anal Calor, 63, 671 (2000)

18) Zhou HY, Wilkes GL, J Mater Sci, 33, 287 (1998).

19) Gordeev SA, Nikolaeva GY, Prokhorov KA, Vysokomolecu Soedin Ser A \& Ser B, 38, 820 (1996).

20) Sheiko SS, Kunz M, Moller M, Vysokomolecu Soedin Ser A \& Ser B, 35, 1903 (1993).

21) Moginger B, Lutz C, Polsak A, Muller U, Colloid Polym Sci, 269, 535 (1991).

22) Oliey RH, Bassett DC, J Macromol Sci-Phys B, 33, 209 (1994).

23) Haward RN, J Polym Sci Part B Polym Phys, 33, 1481 (1995).

24) Gaucher-Miri V, Francois P, Seguela RJ, Polym Sci Part B Polym Phys, 34, 1113 (1996). 
25) Francois $\mathrm{Ph}$, Gaucher V, Seguela R, J Phys Condens Matter, 6, 8959 (1994).

26) Song Y, Shigematsu Y, Nitta K-H, Nemoto N, Polymer J, 34, 584 (2002).

27) Song Y, Nitta K-H, Nemoto N, Macromoleculaes, 36, 1955 (2003).

28) Shigematsu Y, Takada A, Nemoto N, Nitta K-H, Rev Sci Instrum, 72, 3927 (2001).

29) Siesler HW, Adv Polym Sci, 65, 1 (1984).

30) Samuels RJ, Makromol Chem Suppl, 4, 241 (1981).

31) Snyder RG, Schachtschneider JH, Spectrochimica Acta, 20, 853 (1964).

32) Onogi S, Asada T, Takaki T, J Soc Mater Sci Jp, 16, 746 (1967).
33) Bayer G, Hoffmann W, Siesler HW, Polymer, 21, 235 (1980).

34) Masud A, Int J Numer Meth Eng, 47, 1887 (2000).

35) Hutchinson JW, Neale KW, J Mech Phys Solids, 31, 405 (1983).

36) Tomita Y, Hayashi K, Int J Solids Struct, 30, 225 (1993).

37) Khoury FJ, Res Nat Bur St A, 70, 29 (1966).

38) Lin L, Argon AS, J Mater Sci, 29, 294 (1994).

39) Keller A, Pope DP, J Mater Sci, 6, 453 (1971).

40) Porter RS, Wang LH, J Macromol Sci Macromol Chem Phy C, 35, 63 (1995).

41) Peterlin A, Coll Polym Sci, 265, 357 (1987).

42) Pope DP, Keller A, J Polym Sci Polym Phys Edn, 12, 533 (1975). 